\title{
Quorex Pharmaceuticals
}

\author{
New antimicrobials quiet bacterial talk.
}

$$
\text { Pat }
$$
he ability of bacteria to communicate with each other through chemicals was first discovered in the early 1980s. Pathogenic bacteria use this systemso-called quorum sensing - to signal the start of the infection once they have reached a specific density. Several species-specific pathways leading to the release of such chemicals (called autoinducers; Al) were found, but it was not until a signaling system conserved among a range of pathogens-the Al-2 pathway-that researchers saw a means of harnessing quorum sensing for treating bacterial infections. Bonnie Bassler of Princeton University (Princeton, $\mathrm{NJ}$ ) and $\mathrm{M}$ ichael Surette of the University of Calgary (Calgary, Alberta, Canada), who discovered Al-1, founded Quorex Pharmaceuticals in early 1999, along with Jeffrey Stein, then principal scientist at Diversa (San Diego, CA), and Bob Robb, manager of a San Diego-based VC fund, BioVentures West.

Q uorex owns proprietary technologies, gene sequences and proteins (including the Al-2 molecular structure) that may lead to theidentification of new anti-infectives. These drugs are targeted at bacterial surface receptors, preventing them from recognizing autoinducer molecules. This then shuts down the communication pathway that leads to the expression of the pathogen's virulence factors. A secondary result (to be confirmed with additional animal studies) is that the drugs induce a bacteriostatic effect similar to that achieved with chloramphenicol, tetracycline, and erythromycin - the infecting bacteria are unable to obtain nutrients or produce growth factors within the host, and are therefore die or are eliminated by the host's immune system.

New weapons are needed in the fight

Company: Quorex Pharmaceuticals Inc. Founded: 1999

Founders: Bonnie Bassler, Robert Robb (chief executive officer), Jeffrey Stein, and Michael Surette

Employees: 8

Financing to date: $\$ 20.7$ million

Location: Carlsbad, CA

http://www.quorex.com against increasing drug resistance among pathogenic bacteria, caused by the over-use of traditional antibiotics. Quorex's quorumsensing inhibitors could be used against a variety of pathogenic bacteria, including be both more pathogen-specific and less toxic in humans. The company is also looking at non-quorum-sensing targets-newly discovered bacterial proteins that regulate apoptosis in their (mammalian) cell hosts. Bacterial cells control the cell suicide mechanisms of its host to enable their proliferation. This technology and target family was recently acquired from the Burnham Institute (La Jolla, CA).

Another company currently pursuing similar microbial targets is Aurora Biosciences, which acquired Quorum

\section{Quorex has succeeded in attracting an experienced drug development team, led by Krzyzstof Appelt, formerly of Agouron and responsible for the development and commercialization of its HIV drug, Viracept.}

Staphylococcus aureus, Streptococcus pneumoniae, E. coli 0157:H 7 (the cause of recent outbreaks of serious gastroenteritis), $\mathrm{H}$ aemophilus influenzae, and $\mathrm{H}$ eli cobacter pylori. M oreover, because the AI-2 pathway is not prevalent in non-pathogenic bacteria, and is absent in higher organisms, drugs will
Sciences (I owa City, IA) in October 2000. H owever, Quorex has succeeded in attracting an experienced drug development team, led by Krzyzstof Appelt, formerly of Agouron and responsible for the development and commercialization of its HIV drug, Viracept.

\section{Cellular Genomics}

\section{Getting straight to the molecular target.}

wamped with gene sequences, drug companies need fast and accurate ways of validating potential disease targets. In 1998, Yale University's

(New H aven, CT) Ira M ellman perceived that advances in cell biology would be necessary to enable genomic-based drug target discovery and validation, and with the help of Yale's O ffice of Cooperative Research spun off Cellular Genomics.

M ost drug discovery companies use small-molecule combinatorial chemistry or various genetic approaches (e.g., antisense

Founded: 1998

Scientific founders: Ira Mellman, Kevan Shokat, Henrik Dohlman, and Mark Velleca

Employees: 25

Financing to date: $\$ 28$ million

Location: Branford, CT

http://www.cellulargenomics.com

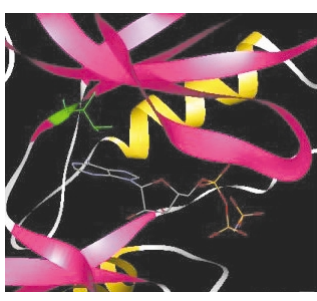

Crystal structure of a kinase with a bound ATP analog.

or gene-knockout technologies) to determine protein function. Louis M atis, Cellular Genomics' president, says that Cellular Genomics brings together the best of both worlds.

The company's proprietary technologies include an analog-sensitive enzyme allele (ASEA) system. The first family of proteins studied using ASEA platform technology are kinases- the enzymes responsible for phosphorylation, and potentially important targets in, for example, cancers and inflammatory disease. Developed by co-founder Kevan Shokat (Nature 407, 395, 2000), ASEA can determine not only the physio- 